\title{
A novel means of comparing the bioequivalence of two formulations of tripotassium dicitrato bismuthate (De-Noltab)
}

\section{S.B. Coghill ${ }^{1}$ and A.N. Shepherd ${ }^{2}$}

${ }^{1}$ Department of Pathology and ${ }^{2}$ Department of Medicine, Ninewells Hospital and Medical School, Dundee, Scotland, UK.

\begin{abstract}
Summary: Tripotassium dicitrato-bismuthate (TDB) has a topical action in the upper gastrointestinal tract. Absorption is minimal and the blood levels are therefore irrelevant to the assessment of bioavailability. TDB is however detectable in the stomach and duodenum by conventional electron microscopy.

Following oral administration of two different formulations of TDB tablets to patients attending a gastroscopy clinic, duodenal biopsies were obtained and examined by electron microscopy. The amount of electron dense drug precipitate was quantified. No difference could be detected between the two formulations.
\end{abstract}

\section{Introduction}

Tripotassium dicitrato-bismuthate (TDP) is effective in the treatment of peptic ulcer disease. ${ }^{1}$ It may be identified in the upper gastrointestinal tract, at its site of action, in both man and rodents by electron microscopy by virtue of the marked electron density of the bismuth in the compound ${ }^{2}$ (Figure 1). Following oral administration of TDB duodenal biopsies have been shown to contain an electron dense precipitate which penetrates the mucus layer

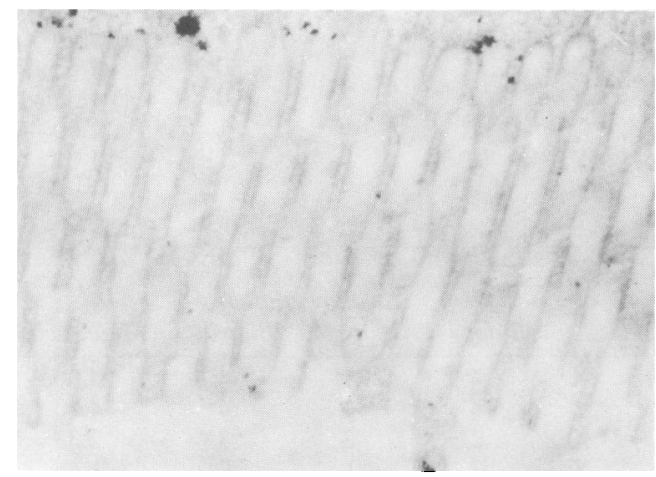

Figure 1 Enterocyte microvilli bearing TDB precipitate (magnification $\times 25,000$ ). Osmium tetroxide post fixation, no counterstain.

Correspondence: S.B. Coghill, B.MSc., M.R.C.Path., Northampton General Hospital, Billing Road, Northampton NN1 5BD, UK.

Accepted: 7 April 1988 and the spaces between microvilli of duodenal enetrocytes. When present in moderate concentrations the precipitate is seen to enter the cells by endocytosis. The drug then accumulates in specialized lysosomes (microsomes) near the apex of the cell (Figure 2). We have never seen the drug penetrate to the lamina propria of normal bowel. This observation correlates well with the relatively low blood bismuth levels achieved with therapeutic levels of TDB when compared to other bismuth compounds which do not form a visible electron dense precipitate. ${ }^{2}$ The precise model of action of

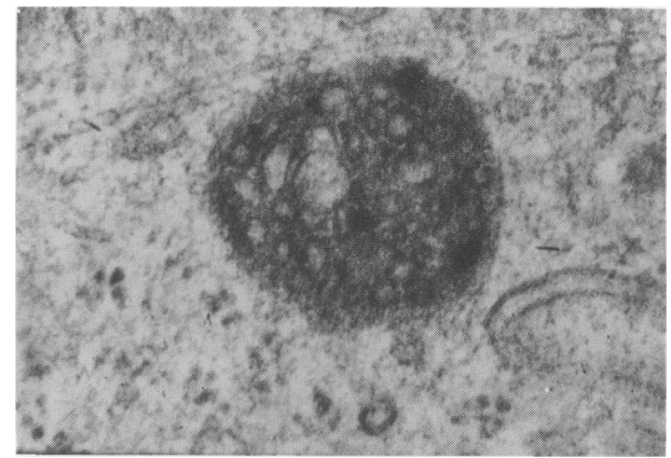

Figure 2 A multivesicular body containing particles of TDB (magnification $\times 60,000$ ). Osmium tetroxide post fixation, no counterstain.

(C) The Fellowship of Postgraduate Medicine, 1988 
TDB is uncertain but evidence of an ulcer coating action has been reported..$^{3,4}$

TDB was originally marketed solely as a liquid (De-Nol, Brocades Great Britain Ltd, Brocades House, Pyrford Road, West Byfleet, Surrey) and then temporarily as a tablet that had to be chewed (De-Noltab). These formulations have previously been compared in a well designed clinical trial. $^{5}$ However, both had characteristics that reduced patient acceptability, notably unpleasant taste with the liquid and blackening of the tongue with the chewing tablet. More recently, to overcome the shortcomings of these preparations, the tablet has been reformulated, to a form that may be swallowed whole.

The trials that show convincingly that TDB is at least as efficacious as other popular, but often more expensive ulcer healing drugs were performed using the older formulations. We have used the electron microscope to compare the quantity of TDB present in the duodenum after administration of the old and the new formulations of De-Noltab.

\section{Patients and methods}

Informed consent was obtained from 10 patients attending the medical out-patient department who were to undergo upper gastrointestinal endoscopy. The patients were receiving no other drugs at the time of the study. Five patients were randomly allocated to receive the chewing tablets and five the swallow tablets. Each patient was supplied with three tablets of either type and asked to take them by chewing or swallowing at mid-day, early evening and on retiring the day before endoscopy. On attending for endoscopy, a fourth tablet was given which was swallowed or chewed 90 minutes before the procedure. Patients in whom an abnormality was identified at endoscopy were excluded from the study and replaced in the stratified randomization.

At endoscopy three biopsies were obtained from separate sites on the duodenum. The tissue was processed conventionally for electron microscopy and ultra-thin, coded sections were examined independently and blind by two observers.

The complete surface of three well orientated villi were examined in each case and the quantity of electron dense TDB precipitate was assessed semiquantitatively on a scale of $0-4$. Electron micrographs were taken and enlarged photographically to give a final magnification of $\times 56,600$. The diameters of one hundred TDB particles were measured in micrographs from each of the ten patients and the mean particle diameter calculated.

\section{Results}

Table I shows the scoring of the two formulations. There was good agreement between the two observers ( $\mathrm{X}$ and $\mathrm{Y})$. Comparison of the two groups of patients shows that although there is some variation in the amount of TDB within the groups, there is no obvious overall difference between the groups.

The mean particle diameter was $35 \mathrm{~nm}$ for the swallow tablet and $32.2 \mathrm{~nm}$ for the chewing tablet with a range of 9-69 for each.

\section{Discussion}

This study suggests that the new formulation of De-Noltab disperses in a similar way to the previously proven formulations and that it appears in the duodenum in similar quantities and in an identical form. It therefore appears reasonable to extrapolate the result of trials on the older formulations to the new one.

Table I The semi-quantitative assessment by two independent observers (X and $\mathrm{Y}$ ), of the amount of electron dense precipitate present on the duodenal microvillous border following the administration of either the chewing or swallowing formulation of De-Noltab.

\begin{tabular}{|c|c|c|c|c|c|}
\hline & & & Chewing & tablets & \\
\hline Patient & & Biopsy 1 & Biopsy 2 & Biopsy 3 & Mean score \\
\hline A & $\mathbf{X}$ & 4 & 3 & 1 & 2.66 \\
\hline & Y & 4 & 4 & 2 & 3.33 \\
\hline B & $\mathbf{X}$ & 4 & 4 & 4 & 4.00 \\
\hline & $\mathbf{Y}$ & 4 & 4 & 4 & 4.00 \\
\hline C & $\mathbf{X}$ & 0 & 3 & 3 & 2.00 \\
\hline & $\mathbf{Y}$ & 0 & 3 & 3 & 2.00 \\
\hline D & $\mathbf{X}$ & 2 & 2 & 0 & 1.33 \\
\hline & $\mathbf{Y}$ & 2 & 2 & 0 & 1.33 \\
\hline E & $\mathbf{X}$ & 1 & 1 & 1 & 1.00 \\
\hline & $\mathbf{Y}$ & 1 & 1 & 1 & 1.00 \\
\hline & & & & & 2.26 \\
\hline
\end{tabular}

(b)

Patient Biopsy 1 Biopsy 2 Biopsy 3 Mean score

\begin{tabular}{llllll}
\hline F & X & 4 & 4 & 4 & 4.00 \\
& Y & 4 & 4 & 4 & 4.00 \\
& X & 2 & 2 & 3 & 2.33 \\
& Y & 2 & 1 & 2 & 1.66 \\
H & X & 4 & 4 & 4 & 4.00 \\
& Y & 4 & 4 & 3 & 3.66 \\
I & X & 1 & 1 & 1 & 1.00 \\
& Y & 1 & 1 & 1 & 1.00 \\
& X & 3 & 2 & 2 & 2.33 \\
& Y & 3 & 3 & 2 & 2.66 \\
& & & & & 2.66 \\
\hline
\end{tabular}


A similar approach to the assessment of the pharmacokinetics and modes of action of TDB and other metal containing drugs, such as sucralphate, may be possible but electron X-ray spectroscopy is likely to be necessary to demonstrate the lighter

\section{References}

1. Editorial. Acid reduction or mucosal protection for peptic ulcer? Lancet 1982, ii: 473-474.

2. Coghill, S.B., Hopwood, D., McPherson, S. \& Hislop, S. The ultrastructural localisation of colloidal tripotassium dicitrato-bismuthate (De-Nol) in the upper gastrointestinal tract of man and rodents following oral and instrumental administration. J Pathol 1983, 139: 105-114.

3. Soutar, R.L. \& Coghill, S.B. Interaction of tripotassium dicitrato-bismuthate with macrophages in the rat and in-vitro. Gastroenterology 1986, 91: 84-93. metal elements such as aluminium. For drugs that are not absorbed and have a coating action in the gastrointestinal tract this type of assessment is likely to be the most realistic assay.

4. Coghill, S.B. Experimental studies using colloidal bismuth subcitrate: possible modes of action. In: Axon, A.T.R. (ed) Pathogenesis and Treatment of Peptic Ulcer Disease. Exerpta Medica, Amsterdam, 1985.

5. Hamilton, I.A. Controlled trial comparing De-Nol Tablets with De-Nol in the treatment of duodenal ulcer. Br Med J 1981, 282: 362. 